\title{
The IC 2118 association: New T Tauri stars in high-latitude molecular clouds
}

\author{
M. Kun ${ }^{1}$, T. Prusti ${ }^{2}$, S. Nikolić ${ }^{3,4}$, L. E. B. Johansson ${ }^{4}$, and N. A. Walton ${ }^{5}$ \\ Konkoly Observatory, 1525 Budapest, PO Box 67, Hungary \\ 2 Astrophysics Missions Division, Research and Scientific Support Department of ESA, Postbus 299, 2200 AG Noordwijk, \\ The Netherlands \\ 3 Astronomical Observatory, Volgina 7, 11160 Belgrade 74, Serbia, Serbia and Montenegro \\ 4 Onsala Space Observatory, 43992 Onsala, Sweden \\ 5 Institute of Astronomy, University of Cambridge, Madingley Road, Cambridge CB3 OHA, UK
}

Received 14 October 2003 / Accepted 21 January 2004

\begin{abstract}
We identified new pre-main sequence stars in the region of high-latitude molecular clouds associated with the reflection nebula IC 2118, around $l \sim 208^{\circ}$ and $b \sim-27^{\circ}$. The stars were selected as T Tauri candidates in objective prism plates obtained with the Schmidt telescope of Konkoly Observatory. Results of spectroscopic follow-up observations, carried out with the FLAIR spectrograph installed on the UK Schmidt and with ALFOSC on Nordic Optical Telescope, are presented in this paper. Based on spectral types, presence of emission lines and lithium absorption line, we identified five classical T Tauri stars and a candidate weak-line T Tauri star projected on the molecular clouds, as well as two candidate pre-main sequence stars outside the nebulous region. Using the near infrared magnitudes obtained from the 2MASS All Sky Catalog (IPAC 2003) we determined the masses and ages of these stars. We found that the five classical T Tauri stars projected on the clouds are physically related to them, whereas the other stars are probably background objects. Adopting a distance of $210 \mathrm{pc}$ for IC 2118 (Kun et al. 2001) and using Palla \& Stahler's (1999) evolutionary tracks we derived an average age of $2.5 \times 10^{6}$ yrs and a mass interval of $0.4-1.0 M_{\odot}$ for the members of the IC 2118 association.
\end{abstract}

Key words. ISM: clouds - ISM: individual objects: IC 2118 - stars: formation - stars: pre-main sequence

\section{Introduction}

Small molecular clouds at high galactic latitudes (Magnani et al. 1985) are usually devoid of star formation. A wellstudied exception is MBM 12, containing the young association of low-mass pre-main sequence (PMS) stars MBM 12A (e.g. Luhman 2001). A less studied example of star forming high latitude molecular cloud is MBM 21 which harbours an infrared source, IRAS 04591-0856, associated with a faint nebulosity HHL 17 (Gyulbudaghian et al. 1987). Persi et al. (1988) have shown that HHL 17 is a low-mass YSO between the protostellar and pre-main sequence evolutionary stage. MBM 21 and 22 are projected at an angular distance of some 10 degrees from the Orion A molecular cloud. They lie at the southernmost part of an extended reflection nebula, IC 2118 (Witch Head Nebula), illuminated by $\beta$ Orionis (Rigel). A ${ }^{12} \mathrm{CO}$ survey performed with the 4-m NANTEN radio telescope and covering the whole area of IC 2118 (Kun et al. 2001, hereinafter Paper I) resulted in the detection of six molecular clouds in the bright region, including MBM 21

Send offprint requests to: $\mathrm{M}$. Kun,

e-mail: kun@konkoly.hu
(G 208.4-28.3) and MBM 22 (G 208.1-27.5). The most massive member of this small group of clouds, G 206.4-26.0, is not included in the MBM catalogue, but was studied by Bally et al. (1991) and Yonekura et al. (1999). Low-mass star formation in G 206.4-26.0 is indicated by the small group of nebulous stars RNO 37 (Cohen 1980), with $\mathrm{H} \alpha$ emission in the two brightest members (Nakano et al. 1995). The northern of these two stars coincides with the IRAS source 05050-0614, having spectral energy distribution indicative of a PMS star (Paper I). Yonekura et al.'s (1999) ${ }^{12} \mathrm{CO},{ }^{13} \mathrm{CO}$ and $\mathrm{C}^{18} \mathrm{O}$ studies have shown that both clouds associated with IRAS point sources contain high-density cores, suitable for forming low-mass stars.

IC 2118 is a part of the Orion region surveyed for weak-line T Tauri stars by Alcalà et al. (1996) on the basis of ROSAT all-sky survey. One wTTS of this sample, RXJ 0502.4-0744 is projected on the reflection nebula, and another one, RXJ 0507.8-0931 is located at about 1.5 degrees to the east of it.

The visual appearance of the clouds associated with IC 2118 suggests their interaction with Orion OB 1 (e.g. Ogura \& Sugitani 1998), therefore they are usually thought to be as distant as the Orion A and B molecular clouds, 
i.e. $\sim 460 \mathrm{pc}$. The radial velocities of the clouds, however, are more negative $\left(-5.30<v_{\mathrm{LSR}}<+4.8 \mathrm{~km} \mathrm{~s}^{-1}\right)$ than both those of the main clouds Orion A and Orion B, $\left(3 \mathrm{~km} \mathrm{~s}^{-1} \leqq v_{\mathrm{LSR}} \leqq\right.$ $11 \mathrm{~km} \mathrm{~s}^{-1}$, Bally 1989; Aoyama et al. 2001) and the bright stars of the Orion OB1 association $\left(v_{\mathrm{LSR}} \sim+5 \mathrm{~km} \mathrm{~s}^{-1}\right.$, Brown et al. 1994). This velocity pattern suggests that whereas the Orion A and B molecular clouds are situated in the receding hemisphere of the expanding interstellar structure around Orion OB1 (Orion-Eridanus Bubble, Brown et al. 1995), the IC 2118 clouds belong to its approaching side. These small clouds therefore are probably closer to us than the expansion centre of the Bubble, Ori OB1a (336 $\pm 16 \mathrm{pc}$, de Zeeuw et al. 1999). Considering the cometary shapes of the clouds, Bally et al. (1991) proposed that they are actually located inside the Bubble, whose radius is about $140 \mathrm{pc}$ (Brown et al. 1994). Based on literature data, Kun et al. (2001) adopted $210 \pm 20 \mathrm{pc}$ for the most probable distance of IC 2118. This result implies that the clouds are situated inside the Orion-Eridanus Bubble, and close to its surface nearest to us.

The age sequence of OB subgroups of Orion OB 1 (e.g. Brown et al. 1994) as well as star formation observed in some cometary globules (e.g. Stanke et al. 2002) suggest that interactions of high-mass stars with the interstellar medium have played a significant role in forming the present appearance of the region. Several observed properties of the IC 2118 region suggest that low mass star formation has been triggered here by the Orion-Eridanus Bubble. We performed a search for additional PMS stars in order to explore the star forming history of the region. Objective prism Schmidt plates were used to search for $\mathrm{H} \alpha$ emission stars, and then spectroscopic follow-up observations of the candidates were carried out in order to establish their nature.

In this paper we present the results of our spectroscopic survey. We describe our observations and data analysis in Sect. 2. Results on the new PMS stars are shown in Sect. 3. A brief summary of the paper is given in Sect. 4. As all of our target stars are included in the 2MASS All Sky Catalog (IPAC 2003), we use the 2MASS source designation for identifying our objects.

\section{Observations}

\subsection{Objective prism observations}

In order to find the possible classical $\mathrm{T}$ Tauri stars in the region of IC 2118 we performed an objective prism Schmidt survey for H $\alpha$ emission stars. Observations were carried out in 1988/1989 at Piszkéstető mountain station of Konkoly Observatory, with the $60 / 90 / 180 \mathrm{~cm}$ Schmidt telescope equipped with a 5 degree objective prism. The field of view of the telescope was a circle of five degrees in diameter. The whole extent of the reflection nebulosity was covered by two, partly overlapping, fields centred on $\alpha(1950)=4^{\mathrm{h}} 58^{\mathrm{m}}, \delta(1950)=-8^{\circ} 20^{\prime}$ and $\alpha(1950)=$ $5^{\mathrm{h}} 4^{\mathrm{m}}, \delta(1950)=-6^{\circ} 30^{\prime}$, respectively. The exposures were taken on Kodak 098-02 and 103a-F emulsions, through an RG1 filter in order to separate the spectral region around the $\mathrm{H} \alpha$ line. The exposure times were 60,72 and $90 \mathrm{~min}$, providing a limiting magnitude of about $R \sim 16$ mag according to our previous studies of this type (Kun 1982, 1986; Kun \& Pásztor 1990). As the region is always seen at large zenith distances from Piszkéstetô ( $47^{\circ} 55^{\prime}$ North), identification of the $\mathrm{H} \alpha$ line was rather uncertain due to the strong sky background. In order not to miss any relevant object, we selected for further study all the dubious cases, 63 stars in all. Equatorial coordinates of the selected objects were computed from their positions, measured on the objective prism plates, using the $\mathrm{H} \alpha$ line as a reference position along the prism spectra. Their $R$ magnitudes were estimated from their diameters in the red POSS prints. As the equatorial coordinates obtained from the objective prism spectra are uncertain to 1-3" (Kun 1982), we used the list of suspected $\mathrm{H} \alpha$ emission stars obtained in this manner together with their finding charts as input data for spectroscopic follow-up observations.

\subsection{Spectroscopy}

Spectroscopic follow-up observations of the PMS star candidates selected from the objective prism observations were carried out at two different epochs and using two different instruments. A sample of 40 stars, brighter than about $R \sim 14.5 \mathrm{mag}$ and distributed over a field of $6^{\circ} \times 6^{\circ}$ centred on RA(1950) $=$ $5^{\mathrm{h}} 0^{\mathrm{m}}$ and $\mathrm{D}(1950)=-8^{\circ} 0^{\prime}$, were observed using the FLAIR multi-fiber spectrograph installed on the UK Schmidt telescope on 15th December, 1993, at poor weather conditions. A pair of frames were taken through the low-dispersion grating G 300B, covering the spectral region between 3800 and $6600 \AA$, and another pair using the high dispersion grating G 1200R, covering the wavelength region $6000-6800 \AA$, each with an exposure time of $3000 \mathrm{~s}$. Domeflat and twilight frames were also obtained for calibration purposes. Neon and Cd-Hg lamp spectra were observed before and after the stellar frames for wavelength calibration. After the standard CCD reduction procedures, performed in IRAF ${ }^{1}$, the images taken through the same grating were coadded. Individual spectra were extracted using the IRAF task "dofibers". The spectral resolution, estimated from the FWHM of the neon lines, was $\lambda / \Delta \lambda \approx 500$ at $\lambda=5000 \AA$ for the $\mathrm{G} 300 \mathrm{~B}$ spectra, and $\lambda / \Delta \lambda \approx 11000$ at $\lambda=6560 \AA$ for the $\mathrm{G} 1200 \mathrm{R}$ spectra. Due to the small angular separation of the components of RNO 37 only its brightest member could be observed during this observing run.

Independently, the PMS star candidates projected on the illuminated clouds were observed using the ALFOSC spectrograph installed on the Nordic Optical Telescope in La Palma in January 2000. The ALFOSC spectra were taken through grism 8 , giving a dispersion of $1.5 \AA /$ pixel over the wavelength region 5800-8350 $\AA$. Using a 1 " slit the spectral resolution was $\lambda / \Delta \lambda \approx 1000$ at $\lambda=6560 \AA$. Spectra of helium and neon lamps were observed before and after each stellar observation for wavelength calibration. We observed a series of spectroscopic standards for spectral classification purposes. We reduced the

${ }^{1}$ IRAF is distributed by the National Optical Astronomy Observatories, which is operated by the Association of Universities for Research in Astronomy, Inc., under contract to the National Science Foundation. 
Table 1. Journal of spectroscopic observations.

\begin{tabular}{lllllllll}
\hline \hline Telescope & Date & Spectrograph Grating/ & $\begin{array}{c}\text { Disp. } \\
\text { Grism }\end{array}$ & $\begin{array}{c}\text { Sp. range } \\
(\AA / \text { pix })\end{array}$ & $\lambda / \Delta \lambda$ & $N$ \\
\hline UK Schmidt & 15 Dec. 1993 & FLAIR & $300 \mathrm{~B}$ & 4.8 & $3800-6600$ & 500 & 40 \\
UK Schmidt & 15 Dec. 1993 & FLAIR & 1200R & 0.35 & $6000-6800$ & 11000 & 40 \\
NOT & 2-7 Jan. 2000 & ALFOSC & Grism 8 & 1.5 & $5825-8350$ & 1000 & 10 \\
\hline
\end{tabular}

Table 2. Results of the spectroscopy and 2MASS data of the stars observed with FLAIR.

\begin{tabular}{clcccrcl}
\hline \hline 2MASS J & Sp. T. & $J$ & $J-H$ & $H-K_{\mathrm{s}}$ & $W(\mathrm{H} \alpha)$ & LiI & Other emission lines \\
\hline $05020630-0850467$ & M0 & 10.897 & 0.782 & 0.441 & $-62.0(5.0)$ & yes & $\mathrm{HeI}, \mathrm{H} \beta,[\mathrm{OI}]$ \\
$05060301-0715472$ & M4 & 11.535 & 0.538 & 0.285 & $-4.1(0.6)$ & no & \\
$05060574-0646151$ & G8: & 11.749 & 0.700 & 0.272 & $-1.87(0.6)$ & yes & \\
$05060913-0712394$ & M3 & 10.888 & 0.557 & 0.246 & $-0.97(0.3)$ & no & \\
$05073060-0610597$ & K7 & 10.127 & 1.081 & 0.743 & $-13.4(1.0)$ & yes & \\
$05094864-0906065$ & G8 & 12.823 & 0.483 & 0.101 & $-4.7(1.0)$ & yes & $\mathrm{H} \beta$ \\
$05112460-0818320$ & M0 & 12.159 & 0.756 & 0.144 & $-30.0(2.0)$ & yes & S[II], [NII] \\
\hline
\end{tabular}

spectra using standard IRAF routines. The journal of the spectroscopic observations is given in Table 1.

\subsection{Spectral classification}

\subsubsection{FLAIR spectroscopy}

The wide wavelength range of FLAIR spectra taken through the grating $300 \mathrm{~B}$ contains several features suitable for spectral classification. Actually the $S / N$ of the blue part of the spectra (at wavelengths shortward of $\mathrm{H} \beta$ ) was too low due to the low sensitivity of the CCD in this spectral region. Useful features for classifying late type stars in the 5000-6600 $\AA$ region are the $\mathrm{MgI}$ lines at 5164-5173, NaI 5890 and 5896, CaI at 6162 and $\mathrm{CaH}$ at $6496 \AA$ for $\mathrm{G}-\mathrm{K}$ types, as well TiO bands at 5167, 5449, 5862 and $6159 \AA$ for the M-type stars. Considering that our $300 \mathrm{~B}$ spectra had nearly the same resolution as the spectrophotometric standards of Pickles' (1998) spectrum library, we utilized this data base in determining the spectral types of the observed stars. After converting the relevant parts of the published data files into IRAF images and normalizing both the observed and standard spectra to the continuum in the same manner, we calibrated several spectral features against the spectral type by measuring their equivalent widths on a grid of standard spectra. The accuracy of the spectral classification is \pm 2 subclasses. Based on the presence of $\mathrm{H} \alpha$ emission and $\mathrm{G}, \mathrm{K}$ or M spectral type seven candidate pre-main sequence stars were identified in the FLAIR G300 B image over the whole field of view of the instrument, five of which were projected on IC 2118. In addition to the above criteria presence of forbidden emission lines as evidence of accretion, and/or strong LiI 6707 absorption line, an important indicator of youth (Bodenheimer 1965) are required for establishing the PMS nature of the stars. It has to be noted as well that the shape and equivalent width of the $\mathrm{H} \alpha$ line in the fiber spectra are uncertain due to the fact that the same average sky spectrum was subtracted from each stellar spectrum, in spite of the variable $\mathrm{H} \alpha$ background throughout the field of view. Therefore presence of the lithium line is a primary criterion of the PMS nature of those target stars which display only weak Balmer emission lines. Spectra taken through the grating G $1200 \mathrm{R}$ have, in principle, sufficiently high resolution to detect the lithium line and separate it from the neighbouring $\mathrm{CaI} \lambda 6718 \AA$ line. Most of our stars have been proved, however, underexposed in the G $1200 \mathrm{R}$ image, and thus the $S / N$ of their spectra was insufficient for measuring reliably the LiI 6707 equivalent width, a key indicator of age for $\mathrm{G}$ and $\mathrm{K}$ type PMS stars. Therefore we used the lithium line as a criterion so that we rejected as PMS objects the stars having $\mathrm{H} \alpha$ emission and no lithium absorption, and classified stars with detected LiI absorption and weak $\mathrm{H} \alpha$ emission as candidate wTTS.

Spectra of the $\mathrm{H} \alpha$ emission stars found during this observing run are shown in Fig. 1. The left panels show the low resolution spectra over the wavelength region $4800-6600 \AA$ and the right panels show the wavelength interval 6540-6740 $\AA$ obtained with the high resolution grating G $1200 \mathrm{R}$. Table 2 shows the results of the FLAIR observations. Spectral types and $\mathrm{H} \alpha$ equivalent widths are given, and we indicate the detection of other emission lines and LiI $\lambda 6707$ absorption in the spectra. $J$ magnitudes, $J-H$ and $H-K_{\mathrm{s}}$ colour indices of the stars from the 2MASS All Sky Catalog (IPAC 2003) are also listed.

According to different classification criteria (e.g. Martín 1997; White \& Basri 2003) for PMS stars, 2MASS J05020630-0850467 and 05073060-0610597 are classical T Tauri stars. The third candidate cTTS is 2MASS J 05112460-0818320, projected far from the IC 2118 molecular clouds. We detected a surprisingly strong variation in the spectrum of this star. The low resolution spectrum shows an early M-type star with weak $\mathrm{H} \alpha$ emission, whereas one can see very strong $\mathrm{H} \alpha$ and other emission features such as 

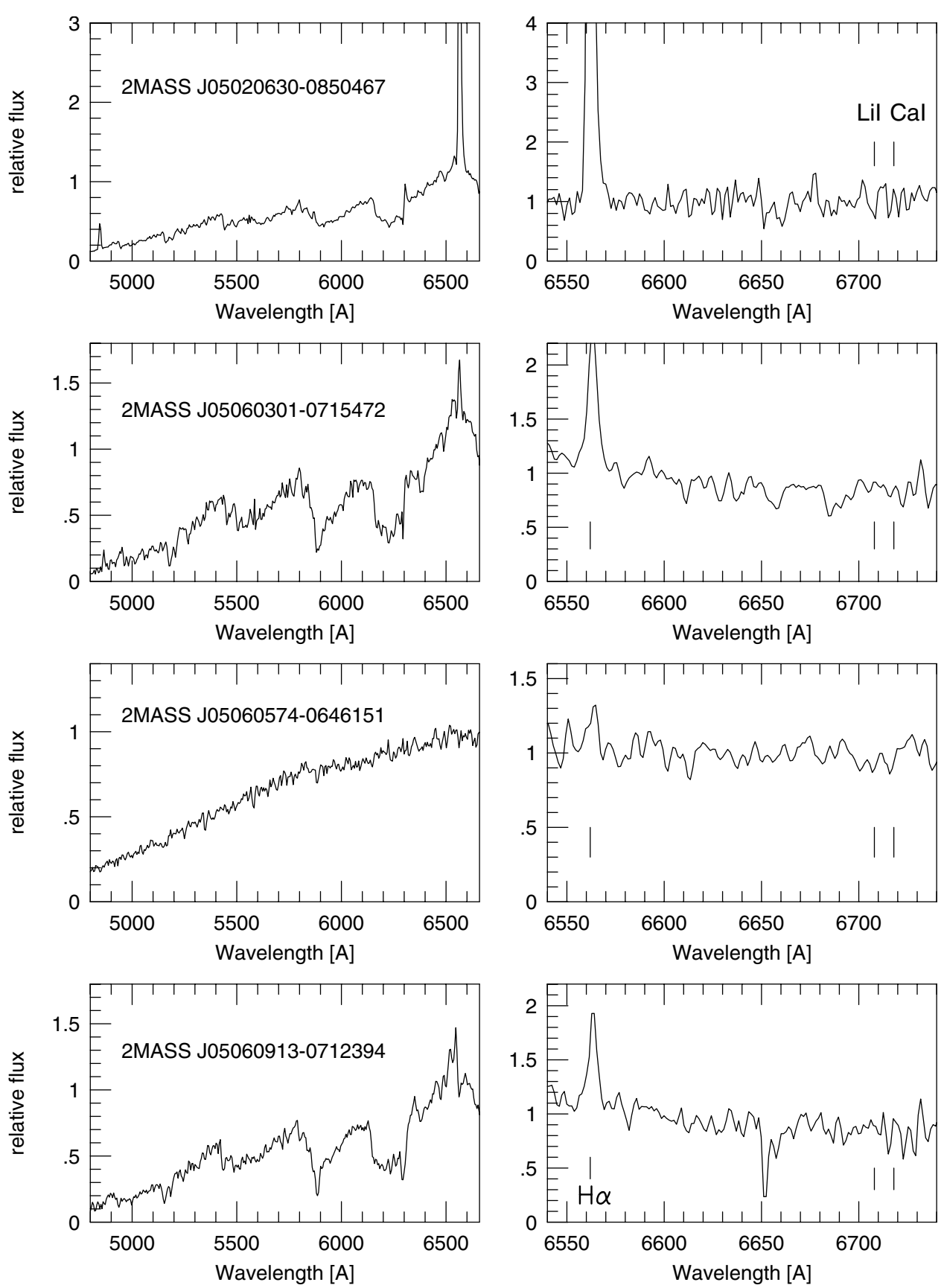

Fig. 1. FLAIR spectra of $\mathrm{H} \alpha$ emission objects found in the region centred on $5^{\mathrm{h}} 0^{\mathrm{m}},-8^{\circ} 0^{\prime}$. Left: low resolution spectra in the wavelength region $4800-6600 \AA$; right: high resolution spectra in the region 6540-6740 ̊. Positions of the H $\alpha$, Li I $\lambda 6707$ and CaI $\lambda 6718$ lines are indicated. No lithium absorption can be seen in the spectra of 05060301-0715472 and 05060913-0712394.

[NII] $\lambda 6584$ and [SII] $\lambda \lambda 6717,6731$ in the high-resolution spectrum, indicating an outburst of the star. Though several features observed in the G1200R spectrum are characteristic of classical $\mathrm{T}$ Tauri stars, the nature of this star remains somewhat uncertain, because it has a position in the $H-K$ vs. $J-H$ colour-colour diagram (see Fig. 4) where PMS stars are unexpected to be found. Unfortunately no photometric data other than the 2MASS is available for this star, although data on photometric variations, accompanying the spectroscopic variation, might be helpful in clarifying the nature of the star and its outburst, as well as could explain its position in the two-colour diagram.
The lithium absorption line is clearly absent from the spectra of 2MASS J05060301-0715472 and J05060913-0712394. The only emission line in the spectra of these M-type stars is $\mathrm{H} \alpha$, indicating that, though they are projected onto the molecular clouds, they are not PMS stars.

2MASS J 05060574-0646151 and 05094864-0906065 are probably weak-line $\mathrm{T}$ Tauri stars, though their nature have to be confirmed with more reliable lithium observations. 2MASS J05060574-0646151 is projected on the molecular cloud $\mathrm{G} 206.4-26.0$, its $\mathrm{H} \beta$ and $\mathrm{H} \alpha$ lines are filled with emission and the LiI $\lambda 6707$ feature can be seen in its high-resolution spectrum. The low resolution spectrum of 

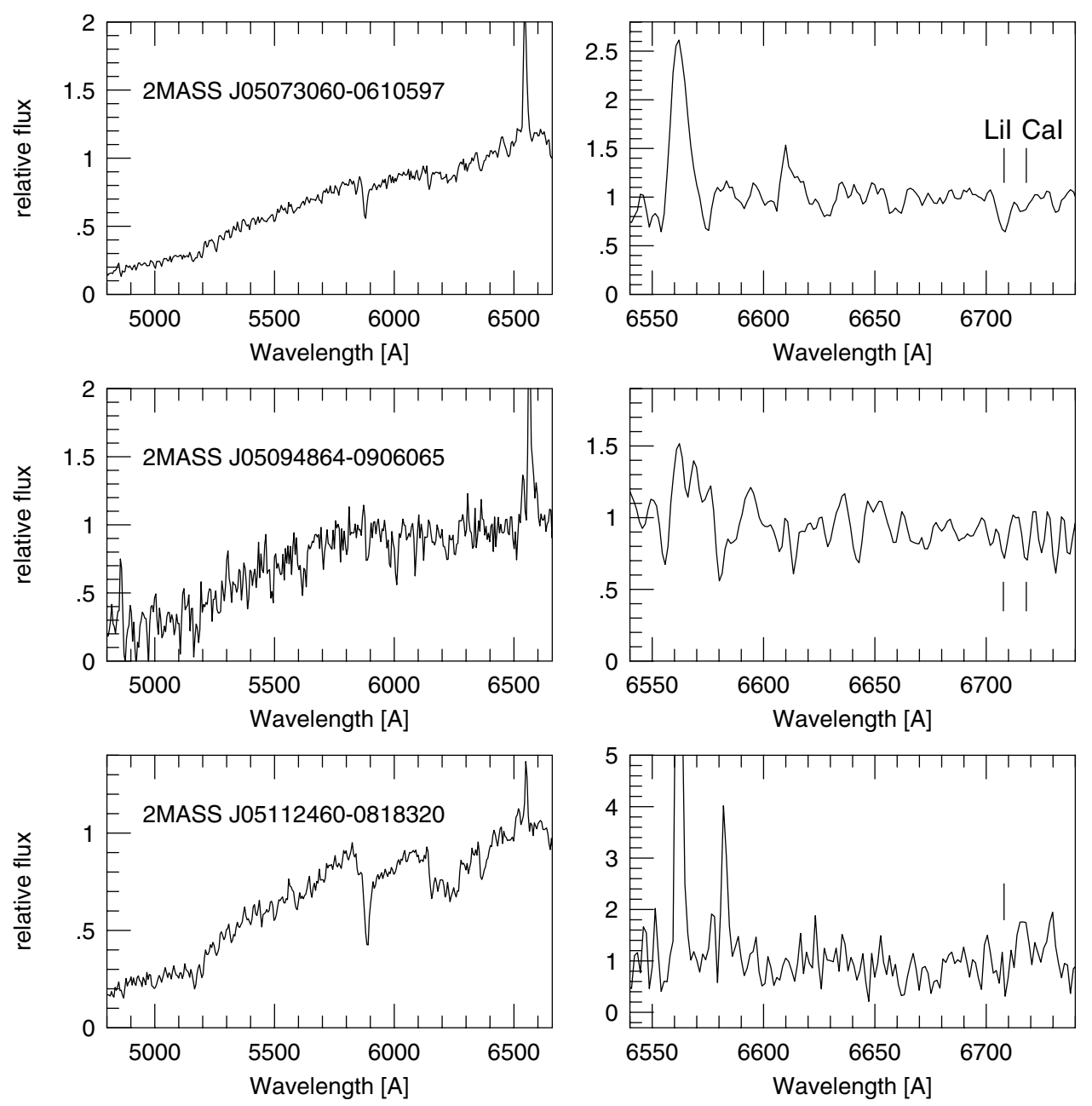

Fig. 1. continued.

2MASS J 05094864-0906065, lying well outside the IC 2118 molecular clouds, displays weak emission in $\mathrm{H} \alpha$ and $\mathrm{H} \beta$, and the $\lambda 6707$ absorption can be recognized in the high-resolution spectrum.

\subsubsection{ALFOSC spectroscopy}

Observations with ALFOSC revealed seven $\mathrm{H} \alpha$ emission objects closely confined to the reflection nebula/molecular clouds. Four of them are common with those observed with FLAIR. Two of the seven, 2MASS J 05060301-0715472 and 05060913-0712394 show neither lithium absorption, nor emission line other than $\mathrm{H} \alpha$. These high $S / N$ observations confirm that they are field stars not related to the clouds on which they are projected. The remaining five stars show both lithium absorption and emission lines characteristic of classical T Tauri stars. Spectra of these objects are shown in Fig. 2. The wavelength range of ALFOSC spectra was suitable for determining several flux ratios defined by Kirkpatrick et al. (1991) ( $A, B$, $C, B / A, B / C)$, Martín \& Kun (1996) $\left(I_{2}, I_{3}\right)$, and Preibisch et al. (2001) $(T 1, T 2)$. We calibrated these spectral features against the spectral type and luminosity class by measuring them in a series of standard stars observed during the same run. The accuracy of the two-dimensional spectral classification, estimated from the range of spectral types obtained from different flux ratios, is \pm 1 subclass, except 2MASS J 05071157-0615098, whose spectrum shows extremely strong emission lines. The flux ratios $I_{2}$ and $I_{3}$ are affected by the wing of the $\mathrm{H} \alpha$ emission line, whereas the wavelength range 7061-7088 in $T_{1}$, contains the HeI emission line at $7065 \AA$, therefore these ratios should not be used. The strong emission spectrum indicates high accretion rate, therefore the effect of the veiling has to be taken into consideration during the spectral classification. The hot continuum excess emission, originating from accretion shocks on the stellar surface, makes the photospheric absorption features shallower than they should be at the given effective temperature. All the spectral features applied during our classification increase in strength with decreasing effective temperature, therefore the effect of the veiling will be an apparently earlier spectral type. Its effect on the measured value of a flux ratio is difficult to quantify, because the broad wavelength intervals compared in the flux ratios contain several faint absorption features beyond the actually measured lines or bands, each affected by the veiling. Veiling is described as a $T=10000 \mathrm{~K}$ blackbody, therefore its contribution is decreasing with increasing wavelength. The spectral type obtained from the flux ratio $A$, measuring the strength of $\mathrm{CaH} \lambda 6975$ feature may be more heavily influenced by veiling 


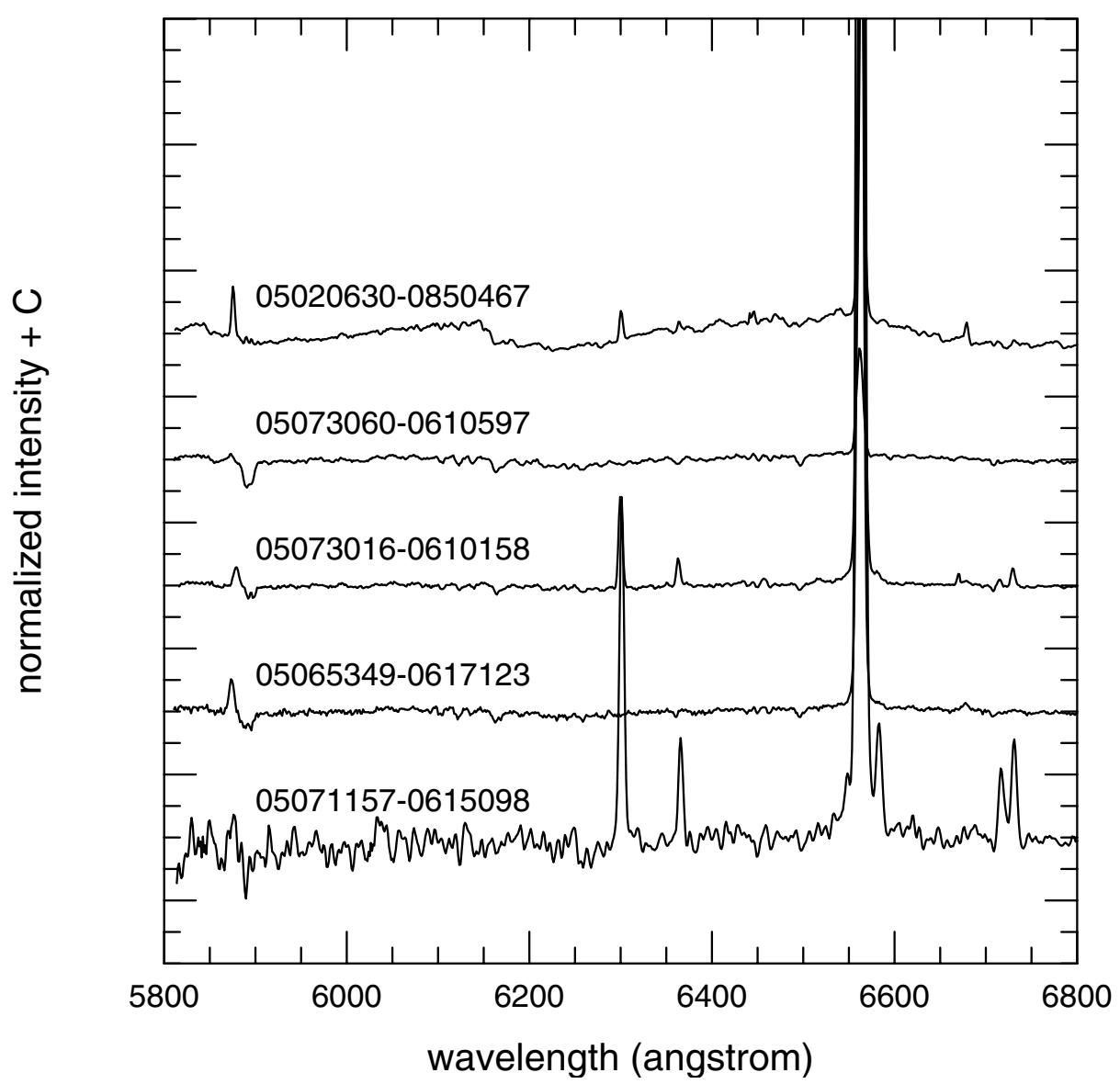

Fig. 2. ALFOSC spectra of T Tauri stars projected on the clouds associated with IC 2118.

Table 3. Pre-main sequence stars associated with IC 2118: results of ALFOSC spectroscopy and 2MASS data.

\begin{tabular}{cllllllll}
\hline \hline 2MASS J & Sp. T. & $J$ & $J-H$ & $H-K_{\mathrm{s}}$ & $W(\mathrm{H} \alpha)$ & $W(\mathrm{LiI})$ & Other emission lines \\
\hline $05020630-0850467$ & M2IV 10.897 & 0.782 & 0.441 & $-58.4(0.8)$ & $0.29(0.03)$ & HeI, NaI \\
$05065349-0617123$ & K7IV & 11.182 & 1.255 & 0.754 & $-112.4(5.0)$ & $0.45(0.03)$ & HeI \\
$05071157-0615098$ & M2IV 13.017 & 1.730 & 1.225 & $-270.0(12.0)$ & $1.05(0.05)$ & [OI], [SII], HeI \\
$05073016-0610158$ & K6IV & 10.839 & 1.254 & 0.963 & $-79.4(1.5)$ & $0.37(0.05)$ & [OI], [NII], [SII], HeI, [FeII] \\
$05073060-0610597$ & K7IV & 10.127 & 1.081 & 0.743 & $-13.5(1.0)$ & $0.46(0.04)$ & HeI \\
\hline
\end{tabular}

than that obtained from the $\mathrm{NaI} \lambda \lambda 8183,8195$ lines (flux ratio $C$ ). We found no systematic difference between the spectral types derived from $A, B, C, B / A, B / C$. The range of the derived spectral types resulted in the accuracy of \pm 2 subclasses for 2MASS J 05071157-0615098.

Results of spectroscopy of PMS stars performed with ALFOSC are presented in Table 3 . In addition to the derived spectral types we present the equivalent widths of the $\mathrm{H} \alpha$ and LiI lines, as well as indicate the additional emission lines observed in the spectra. The uncertainties given in parentheses have been derived from the repeatability of the measurements. The real uncertainties of the LiI equivalent widths may be higher due to the blending of the line with neighbouring absorption or emission features (CaI $\lambda 6718$, [SII] 26717 ). $J$ magnitudes, $J-H$ and $H-K_{\mathrm{s}}$ colour indices from the 2MASS
All Sky Catalog (IPAC 2003) are also shown. Both Fig. 2 and Table 3 clearly show that all these stars are cTTS.

\section{Discussion}

Figure 3 shows the surface distribution of the newly found PMS stars, together with other known YSOs of the region, overlaid on the IRAS $100 \mu \mathrm{m}$ image. In addition to the objects listed in Tables 2 and 3 two wTTS, identified by Alcalà et al. (1996), and the embedded YSO IRAS 04591-0856 are plotted. The five cTTS listed in Table 3, IRAS 04591-0856, as well as the candidate wTTS 2MASS J 05060574-0646151 are projected on the molecular clouds associated with IC 2118, while RXJ 0502.4-0744 is projected against a lower density part of the cloudy region. 


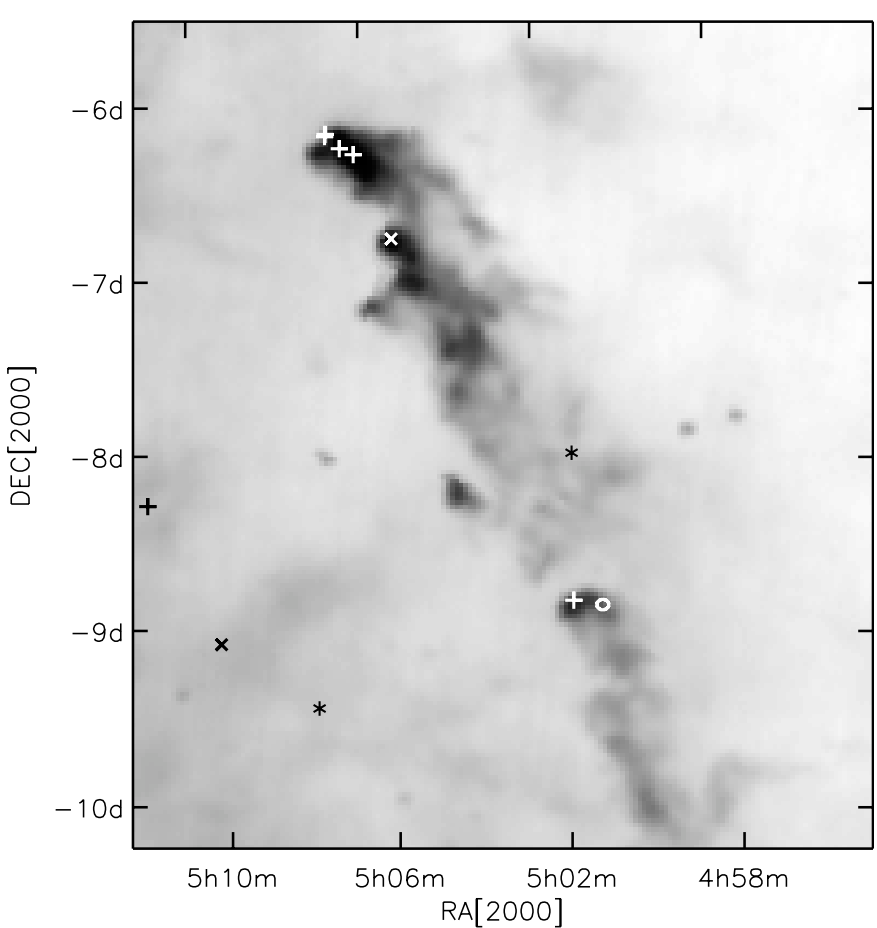

Fig. 3. Distribution of the new PMS stars and other young stellar objects overlaid on the $100 \mu \mathrm{m}$ IRAS image of the region. "+" signs mark the new classical T Tauri stars (including the candidate cTTS 2MASS J 05112460-0818320), and the candidate wTTS found during the present survey are marked with " $\times$ ". The open circle shows the position of IRAS 04591-0856 (HHL 17). Asterisks mark the weak-line T Tauri stars identified by Alcalà et al. (1996) in the ROSAT all-sky survey data base.

\subsection{HRD of the target stars}

We used $J, H$, and $K_{\mathrm{s}}$ magnitudes obtained from the 2MASS All Sky Catalog (IPAC 2003) to place our stars on the Hertzsprung-Russell diagram. For this purpose their effective temperatures and bolometric luminosities are to be determined. $T_{\text {eff }}$ comes from the spectral type (Kenyon \& Hartmann 1995), whereas $L_{\text {bol }}$ can be determined from the near-infrared photometric data. Figure 4 displays their positions on the $H-K_{\mathrm{s}}$ vs. $J-H$ colour-colour diagram together with the lines indicating the position of zero-age main-sequence, the giant branch, direction of the interstellar reddening and the locus of classical $\mathrm{T}$ Tauri stars determined by Meyer et al. (1997). In addition to the stars found during the present survey, the ROSAT wTTS (Alcalà et al. 1996) are also plotted.

The four cTTS associated with the cloud G 206.4-26.0, and HHL 17 clearly display infrared excess, located to the right of the band of the reddened main sequence, whereas the positions of 2MASS J 05020630-0850467, associated with the cloud G 208.4-28.3 and 2MASS J05060574-0646151, projected on G206.8-26.5, are equally compatible with unreddened cTTS and reddened main sequence stars. 2MASS J05112460-0818320, which displayed the outburst during the FLAIR observing run, is also marked as cTTS in Fig. 4, though it lies on the giant sequence, rendering its nature somewhat uncertain.

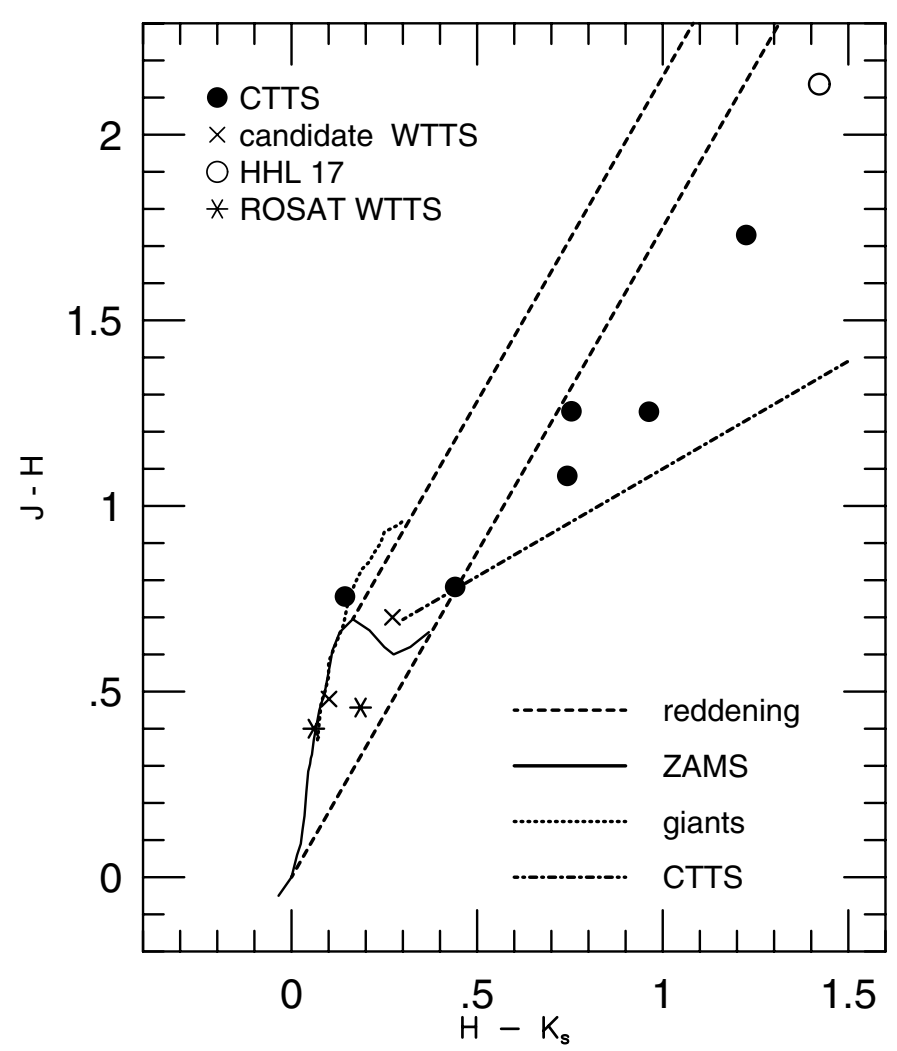

Fig. 4. Positions of the PMS stars in IC 2118 in the $J-H$ vs. $H-K_{\mathrm{s}}$ diagram. Loci of zero-age main sequence, giant branch, and classical T Tauri stars, as well as the slope of interstellar reddening are indicated. 2MASS J05112460-0818320, displaying cTTS-like spectrum in the G 1200R image, is located on the giant branch.

We made the widely used assumption that the total emission of our target stars in the $J$ band originates from the photosphere (e.g. Hartigan et al. 1994). Thus the colour index $J-H$ can be written as

$J-H=(J-H)_{0}+E_{\mathrm{CS}}(J-H)+E_{\mathrm{IS}}(J-H)$,

where $(J-H)_{0}$ is the true photospheric colour of the star, $E_{\mathrm{CS}}(J-H)$ is the colour excess due to the emission from the circumstellar disk in the $H$ band, and $E_{\mathrm{IS}}(J-H)$ is the colour excess originating from the difference of interstellar extinctions in the $J$ and $H$ bands.

We dereddened our cTTS onto the locus of unreddened T Tauri stars in the $H-K_{\mathrm{s}}$ vs. $J-H$ colour-colour diagram (Meyer et al. 1997) in order to determine $E_{\mathrm{IS}}(J-H)$. Bolometric luminosities were derived from the $J$ magnitudes and $E_{\mathrm{IS}}(J-H)$ colour excesses by using the interstellar extinction law $A_{\mathrm{J}}=2.65 \times E_{\mathrm{IS}}(J-H)$ (Rieke \& Lebofsky 1985), and the bolometric corrections tabulated by Hartigan et al. (1994).

The distribution of our target stars in the HRD is displayed in Fig. 5, with the assumption that all of them are located at $460 \mathrm{pc}$, at the distance of main Orion molecular clouds. Uncertainties of $\log T_{\text {eff }}$ are derived from those of the spectral classification. In assessing the uncertainty of $\log L$ the errors of photometric data, given in the 2MASS All Sky Catalog, and uncertainties of the bolometric corrections due to the error of spectral classification were taken into account. Further 
Table 4. Properties of the pre-main sequence stars associated with IC 2118, derived from spectroscopic and 2MASS data.

\begin{tabular}{cccccc}
\hline \hline 2MASS J & $\begin{array}{c}T_{\text {eff }} \\
(\mathrm{K})\end{array}$ & $\begin{array}{c}A_{\mathrm{V}} \\
(\mathrm{mag})\end{array}$ & $\begin{array}{c}L \\
\left(L_{\odot}\right)\end{array}$ & $\begin{array}{c}M \\
\left(M_{\odot}\right)\end{array}$ & $\begin{array}{c}\text { Age } \\
\left(10^{6} \mathrm{yr}\right)\end{array}$ \\
\hline $05020630-0850467$ & $3580\left(\begin{array}{c}3720 \\
3470\end{array}\right)$ & $0.0\left(\begin{array}{c}+0.5 \\
-0.5\end{array}\right)$ & $0.33\left(\begin{array}{c}0.42 \\
0.27\end{array}\right)$ & $0.38\left(\begin{array}{c}0.45 \\
0.30\end{array}\right)$ & $1.5\left(\begin{array}{c}2.0 \\
1.0\end{array}\right)$ \\
$05065349-0617123$ & $4060\left(\begin{array}{c}4205 \\
3955\end{array}\right)$ & $4.0\left(\begin{array}{c}4.7 \\
3.4\end{array}\right)$ & $0.84\left(\begin{array}{l}1.04 \\
0.66\end{array}\right)$ & $0.80\left(\begin{array}{c}0.90 \\
0.66\end{array}\right)$ & $2.5\left(\begin{array}{c}3.0 \\
2.0\end{array}\right)$ \\
$05071157-0615098$ & $3580\left(\begin{array}{c}3850 \\
3370\end{array}\right)$ & $6.8\left(\begin{array}{c}7.8 \\
6.3\end{array}\right)$ & $0.29\left(\begin{array}{c}0.36 \\
0.23\end{array}\right)$ & $0.38\left(\begin{array}{c}0.45 \\
0.22\end{array}\right)$ & $2.0\left(\begin{array}{l}3.0 \\
0.9\end{array}\right)$ \\
$05073016-0610158$ & $4205\left(\begin{array}{c}4350 \\
4060\end{array}\right)$ & $2.5\left(\begin{array}{c}3.1 \\
2.1\end{array}\right)$ & $0.77\left(\begin{array}{c}0.93 \\
0.63\end{array}\right)$ & $0.90\left(\begin{array}{c}1.05 \\
0.80\end{array}\right)$ & $4.0\left(\begin{array}{c}5.5 \\
2.5\end{array}\right)$ \\
$05073060-0610597$ & $4060\left(\begin{array}{c}4205 \\
3955\end{array}\right)$ & $1.7\left(\begin{array}{c}2.4 \\
1.2\end{array}\right)$ & $1.23\left(\begin{array}{c}1.56 \\
1.00\end{array}\right)$ & $0.80\left(\begin{array}{c}0.90 \\
0.66\end{array}\right)$ & $1.0\left(\begin{array}{c}1.6 \\
0.9\end{array}\right)$ \\
\hline
\end{tabular}

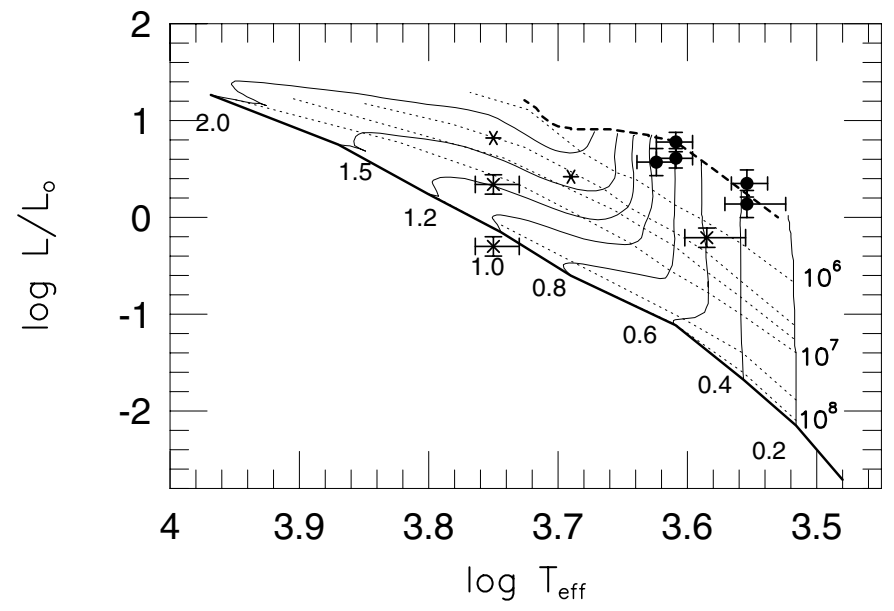

Fig. 5. Positions of the observed stars in the HRD, assuming a distance of $460 \mathrm{pc}$. Black dots indicate classical T Tauri stars associated with IC 2118, crosses mark the other target stars and asterisks are for wTTS detected by ROSAT (Alcalà et al. 1996). Dotted lines indicate the isochrones of $10^{6}, 3 \times 10^{6}, 5 \times 10^{6}, 10^{7}, 5 \times 10^{7}$ and $10^{8}$ years, and thin solid lines show the evolutionary tracks from Palla \& Stahler's (1999) model. The dashed line corresponds to the birthline and thick solid line indicates the zero age main sequence.

sources of uncertainty of $\log L$ are negligence of the excess luminosity arising from photospheric veiling and circumstellar dust emission. Both of these effects, however, have their minima around $1 \mu \mathrm{m}$ (Kenyon \& Hartmann 1995). Evolutionary tracks and isochrones, as well as the position of the birthline and zero-age main sequence (Palla \& Stahler 1999) are also shown. However, distances of the stars, in particular of those outside the IC 2118 molecular clouds, are actually unknown. They may either be low-mass members of Orion OB1, closer to us than the A and B clouds, or may be situated at different distances as members of the Gould Belt system (Alcalà et al. 1998). Therefore this figure only indicates that if they are as distant as the giant clouds of most recent star formation, then most of them are PMS stars at different evolutionary stages. The only exception is 2MASS J 05094864-0906065, located far from the IC 2118 clouds on the sky, and below the ZAMS in Fig. 5. This star is probably more distant $(d \gtrsim 800 \mathrm{pc})$ than the Orion star forming region, given that $\mathrm{H} \alpha$ and $\mathrm{H} \beta$ emission, seen in its spectrum, are indicative of PMS nature for this spectral type. In this figure the five cTTS found in the IC 2118 molecular clouds are located high above the $1 \mathrm{Myr}$ isochrone,

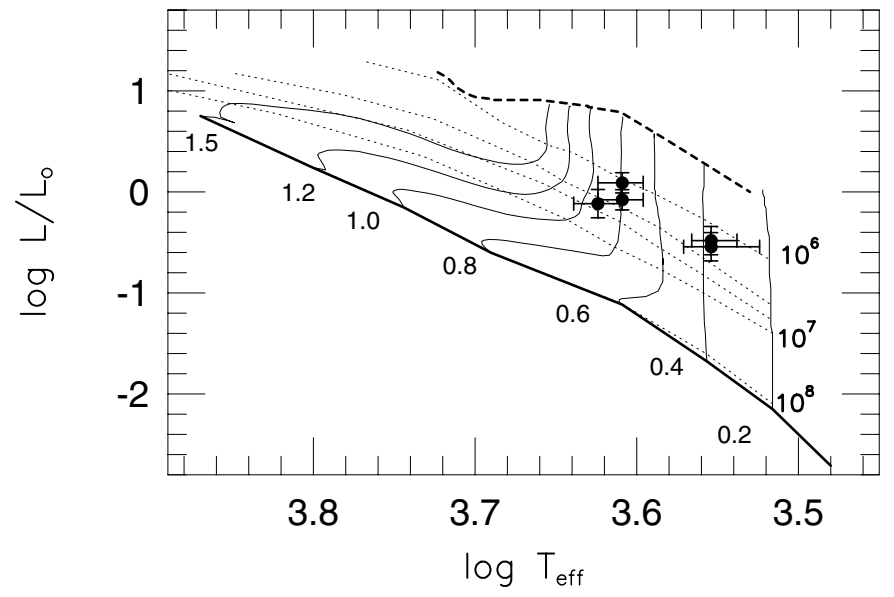

Fig. 6. The T Tauri stars of IC 2118 in the HRD, assuming a distance of $210 \mathrm{pc}$. Isochrones and evolutionary tracks, as well as the birthline and zero age main sequence are indicated as in Fig. 5.

around the birthline. It was shown by Baraffe et al. (2002) that stellar ages and evolutionary tracks are very uncertain at this part of the HRD. The birthline shown here is considered as an upper limit for pre-main sequence luminosities, and even it is probable that the youngest accreting low-mass stars appear below this line (Hartmann et al. 1997). Four of the five cTTS are located at the outer regions of the dense $\mathrm{C}^{18} \mathrm{O}$ cores of their parent clouds (Yonekura et al. 1999), suggesting that they have already evolved off the birthline. Therefore their positions in this diagram provide further support for the result that IC 2118 is closer to us than Orion A and B.

Adopting that IC 2118 is located at a distance $210 \mathrm{pc}$ from us, the HRD shown in Fig. 6 has been obtained. In this plot the cTTS projected on IC 2118 form a group in the mass interval $0.4-0.9 M_{\odot}$, and they are scattered between the isochrones of $1 \times 10^{6}$ and $4 \times 10^{6}$ years. The candidate wTTS seen along the line of sight of a molecular cloud, 2MASS J 0506057-0646151, is probably a more distant object, lying behind the clouds. Properties of these five $T$ Tauri stars, resulted from our study, are shown in Table 4. Effective temperatures $T_{\text {eff }}$ corresponding to the spectral classes are displayed in Col. 2, and visual extinctions $A_{\mathrm{V}}=9.14 \times E_{\mathrm{IS}}(J-H)$ (Rieke \& Lebofsky 1985) are shown in Col. 3. Luminosities derived from the 2MASS data are listed in Col. 4, and masses and ages resulting from the Palla \& Stahler (1999) model, 
are shown in Cols. 5 and 6, respectively. Minimum and maximum values of the derived quantities, resulting from the errors of spectral classification and photometry, are indicated in parentheses.

\subsection{The IC 2118 association}

Both the surface distribution of the newly identified cTTS and their position in the HRD suggest the presence of a young association of low-mass stars formed in the high latitude molecular clouds associated with IC 2118. The five cTTS identified in this work are projected on two different molecular clouds. The cloud G 206.4-26.0 hosts four of the stars. The mass of this cloud is $85 M_{\odot}$ (Paper I), and its radial velocity of $v_{\text {LSR }} \approx-2.2 \mathrm{~km} \mathrm{~s}^{-1}$, significantly more negative than those of Orion $\mathrm{OB} 1$ and Orion $\mathrm{A}$ and $\mathrm{B}$, suggesting that it represents a distinct subsystem of the Orion star forming region. It contains an elongated dense core mapped in $\mathrm{C}^{18} \mathrm{O}$ by Yonekura et al. (1999). The mass of the cores, traced by $\mathrm{C}^{18} \mathrm{O}$, is $25 M_{\odot}$ (scaled to 210 pc Yonekura et al.'s (1999) result). The stars associated with this cloud are aligned parallel to the long axis of the core, at a mean projected distance of $\sim 0.3 \mathrm{pc}$ from each other, so that the two nebulous objects in RNO 37 as well as $05065349-0617123$ are located at the outskirts, while the 05071157-0615098 is projected inside the core (see Fig. 3: the IRAS $100 \mu \mathrm{m}$ intensities show largely the same structure as ${ }^{13} \mathrm{CO}$ and $\mathrm{C}^{18} \mathrm{O}$ maps). This surface distribution suggests an age sequence: the star closer to the centre of the core should be younger. This age sequence is washed out by the uncertainties of $T_{\text {eff }}$ and $L_{\mathrm{bol}}$, but the signposts of strong accretion, observed in the spectrum of 05071157-0615098 may be indicative of its extreme youth indeed. This star may be significantly younger than the age derived from its position in the HRD. Both theoretical and observational studies suggest that strongly accreting PMS stars may be considerably less luminous than their coeval, non-accreting counterparts, mimicking an older age (Hartmann et al. 1997; Comerón et al. 2003).

Comparison of the $J$ magnitudes of the 2MASS and DENIS (DENIS Consortium 2003) data bases, moreover, reveals the variability of this star: contrary to the 2MASS magnitude $J=13.017 \pm 0.026$, the same value for DENIS J 050711.5-061509 is $J=12.693 \pm 0.08 \mathrm{mag}$. The variability in the $J$ band also contributes to the uncertainty of the derived luminosity.

The fifth member of the IC 2118 association, 2MASS J05020630-0850467 is projected on the molecular cloud G208.3-28.4 (MBM 21), whose mass was estimated to be $14 M_{\odot}$ (Paper I). The radial velocity of this cloud is $v_{\mathrm{LSR}}=+4.8 \mathrm{~km} \mathrm{~s}^{-1}$, close to the average value of Orion OB1. The large velocity difference between the northern and southern clouds of the IC 2118 complex may suggest that they are unrelated objects at different distances. This is unlikely because both the illumination of the northern clouds by Rigel and the distance determination for the southern cloud by Penprase (1993) converge to the same distance value adopted here. One may notice, however, that the velocity pattern of the IC 2118 complex is similar to that observed by Bally (1989) in Orion A: while the radial velocities of the southern parts of both Orion A and IC 2118 are nearly the same as that of Orion $\mathrm{OB} 1$, the northern portions have more positive velocities in Orion $\mathrm{A}$ and more negative velocities in IC 2118. Both regions are located to the south of Ori OB1a, the centre of the expansion of the Orion-Eridanus Bubble; Orion A resides in the receding hemisphere, and IC 2118 in the approaching one. Thus the observed velocity structures suggest that the northern parts of the clouds, closer to Ori OB1, have experienced greater acceleration than those farther from the origin of the shock wave, compressing and subsequently accelerating the clouds.

The cloud contains two dense $\mathrm{C}^{18} \mathrm{O}$ cores having masses of 7.7 and $3.5 M_{\odot}$, respectively (scaled to $210 \mathrm{pc}$ the values derived by Yonekura et al. 1999). The star is located at the edge of the larger, eastern core, whereas the smaller, western core contains the embedded infrared source IRAS 04591-0856.

The large-scale geometry and kinematics of the OrionEridanus region suggests that star formation in the IC 2118 region propagates from the north-east toward the south-west, and also toward us. According to this picture, the two YSOs associated with G 208.3-28.4 are probably somewhat younger than their counterparts in G 206.4-26.0. Both the derived age of J 05020630-0850467 and the deeply embedded state of IRAS 04591-0856 support this hypothesis.

G 208.3-28.4 is one of the smallest known star forming molecular clouds in our galactic neighbourhood. Its star forming cores contain significantly less material than the average $\mathrm{C}^{18} \mathrm{O}$ mass of $\approx 12 M_{\odot}$, required to form a protostar in Taurus and Chamaeleon (Onishi et al. 1999; Mizuno et al. 1999). This may be the consequence of the high ambient pressure from the superbubble, leading to a smaller critical mass for gravitational collapse.

The velocity gradient along the cloud complex suggests that the compressed clouds are subsequently accelerated by the shock propagating from Ori OB1, and that their acceleration continues after the onset of star formation. The probability of observing YSOs in very small, compressed clouds is low not only because small clouds disperse rapidly, but also because they are swept off the newly formed stars, which keep their velocities while their parental clouds are further accelerated.

Alcalà et al. (2000) identified a subsample of wTTS widely distributed over the Orion region with radial velocities $v_{\text {LSR }}<$ $+6 \mathrm{~km} \mathrm{~s}^{-1}$, among them are the two stars, RX J0502.4-0744 and RX J0507.8-0931, located within the field studied here. The presence of the young stars in G 208.3-28.4 gives some support to the speculation that these stars might have been born in small clouds compressed to form stars and then swept aside and dispersed by the approaching hemisphere of the superbubble. In this case the distance of these stars should be between 200-350 pc. The positions in the HRD of both RXJ0502.4-0744 and RXJ0507.8-0931 favour the higher limit, because, according to the scenario of sequential star formation, they should be younger than $10^{7}$ years.

It was established in Paper I that the clouds associated with IC 2118 lie on the surface of the Orion-Eridanus Bubble, being blown with variable powers by the stellar winds and supernova explosions of the massive stars of Orion OB1 during the last ten million years. The ages of the PMS stars found in the clouds are 
compatible with the assumption that star formation has been triggered by the superbubble. The complicated geometry and wind history of the OB association (Brown et al. 1995) hinders both any detailed speculation on the exact position and age of the sources of trigger and any accurate mapping of the shape of the bubble surface. Wherever the shock wave meets a dense medium, a new section of surface will arise.

The stars found during the present studies are probably the most massive members of the young stellar group born in the low-mass, high-latitude molecular clouds. Several faint and red 2MASS and DENIS sources are projected on the clouds, whose nature is uncertain due to the low $S / N$ of the data. Further members of the IC 2118 association can be revealed by spectroscopic and deep near infrared observations of these sources.

\section{Summary of results}

We identified a new nearby association of classical T Tauri stars in the region of the reflection nebula IC 2118, at a distance of $210 \mathrm{pc}$ from the Sun. Our spectral classification, together with near-infrared photometric data published in the 2MASS All Sky Catalog (IPAC 2003), allowed us to derive effective temperatures and luminosities of these stars. Comparison of these data with theoretical pre-main sequence evolutionary tracks has shown that masses of the association members are in the interval $0.4-0.9 M_{\odot}$, and their ages are in the interval $1-4 \mathrm{Myr}$. Our results suggest that star formation was triggered in the IC 2118 clouds by shock waves originating from Orion OB 1 , and thus this group of young low-mass stars is a distinct subsystem of the Orion star forming region.

Acknowledgements. We are indebted to Quentin Parker and Paul Cass for their help in obtaining FLAIR data, and to Francesco Palla for sending his data set on pre-main sequence evolution. This work is partly based on observations with Nordic Optical Telescope operated on the island of La Palma jointly by Denmark, Finland, Iceland, Norway, and Sweden, in the Spanish Observatorio del Roque de los Muchachos of the Instituto de Astrofisica de Canarias. The data presented here have been taken using ALFOSC, which is owned by the Instituto de Astrofisica de Andalucia (IAA) and operated at the Nordic Optical Telescope under agreement between IAA and the NBIfAFG of the Astronomical Observatory of Copenhagen. This publication makes use of data products from the Two Micron All Sky Survey, which is a joint project of the University of Massachusetts and the Infrared Processing and Analysis Center/California Institute of Technology, funded by the National Aeronautics and Space Administration and the National Science Foundation. We also utilized DENIS data DENIS has been supported financially mainly by the French Institut National des Sciences de l'Univers, CNRS, and French Education Ministry, the European Southern Observatory, the State of Baden-Wuerttemberg, and the European Commission under networks of the SCIENCE and Human Capital and Mobility programs, the Landessternwarte, Heidelberg, l'Institut d'Astrophysique de Paris, the Institut für Astrophysik der Universität Innsbruck and Instituto de Astrofisica de Canarias.

Financial support from the Hungarian OTKA grants T34584 and T37508, and from the Serbian P1191 grant is acknowledged. The paper is benefited from the comments of the anonymous referee.

\section{References}

Alcalà, J. M., Terranegra, L., Wichmann, R., et al. 1996, A\&AS, 119, 7

Alcalà, J. M., Chavarría, K. C., \& Terranegra, L. 1998, A\&A, 330, 1017

Alcalà, J. M., Covino, E., Torres, G., et al. 2000, A\&A, 353, 186

Aoyama, H., Mizuno, N., Yamamoto, H., et al. 2001, PASJ, 53, 1053

Bally, J. 1989, Low Mass Star Formation and Pre-Main Sequence Objects, ed. B. Reipurth, ESO Conference and Workshop Proc., 33,1

Bally, J., Langer, W. D., Wilson, R. W., et al. 1991, Fragmentation of Molecular Clouds and Star Formation, IAU Symp., 147, ed. E. Falgarone, F. Boulanger, \& G. Duvert (Kluwer), 11

Baraffe, I., Chabrier, G., Allard, F. \& Hauschildt, P. H. 2002, A\&A, 382,563

Bodenheimer, P. 1965, ApJ, 141, 451

Brown, A. G. A., de Geus, E. J., \& de Zeeuw, P. T. 1994, A\&A, 289, 101

Brown, A. G. A., Hartmann, D., \& Burton, W. 1995, A\&A, 300, 903

Cohen, M. 1980, AJ, 85, 29

Comerón, F., Fernández, I., Baraffe, I., Neuhäuser, R., \& Kaas, A. A. 2003, A\&A, 406, 1001

The DENIS Consortium 2003, http://cdsweb.u-strasbg.fr/denis.html

de Zeeuw, P. T., Hoogerwerf, R., de Bruijne, H. J., Brown, A. G. A., \& Blaauw A. 1999, AJ, 117, 354

Gyulbudaghian, A. L., Rodríguez, L. F., \& Mendoza-Torres, E. 1987, Rev. Mex. Astron. Astrofis., 15, 53

Hartigan, P., Strom, K. M., \& Strom, S. E. 1994, ApJ, 427, 961

Hartmann, L., Cassen, P., \& Kenyon, S. J. 1997, ApJ, 475, 770

IPAC 2003, The 2MASS All Sky Catalog, www. ipac. caltech. edu

Kenyon, S. J., \& Hartmann, L. 1995, ApJS, 101, 117

Kirkpatrick, J. D., Henry, T. J., \& McCarthy, D. W. 1991, ApJS, 77, 417

Kun, M. 1982, Afz, 18, 37

Kun, M. 1986, Ap\&SS, 125, 13

Kun, M., \& Pásztor L. 1990, Ap\&SS, 174, 13

Kun, M., Aoyama, H., Yoshikawa, N., et al. 2001, PASJ, 53, 1063 (Paper I)

Luhman, K. L. 2001, ApJ, 560, 287

Magnani, L., Blitz, L., \& Mundy, L. 1985, ApJ, 295, 402

Martín, E. L. 1997, A\&A, 321, 492

Martín, E. L., \& Kun, M. 1996, A\&AS, 116, 467

Meyer, M. R., Calvet, N., \& Hillenbrand, L. A. 1997, AJ, 114, 288

Mizuno, A., Hayakawa, T., Tachihara, K., et al. 1999, PASJ, 51, 859

Nakano, M., Wiramihardja, S. D., \& Kogure, T. 1995, PASJ, 47, 889

Ogura, K., \& Sugitani, K. 1998, Publ. Astron. Soc. Aust., 15, 91

Onishi, T., Mizuno, A., Kawamura, A., et al. 1998, ApJ, 502, 296

Palla, F., \& Stahler, S. W. 1999, ApJ, 525, 772

Penprase, B. E. 1993, ApJS, 88, 433

Persi, P., Ferrari-Toniolo, M., Busso, M., et al. 1988, AJ, 95, 1167

Pickles, A. J. 1998, PASP, 110, 863

Preibisch, T., Guenther, E. \& Zinnecker, H. 2001, AJ, 121, 1040

Rieke, G. H., \& Lebofsky, M. J. 1985, ApJ, 288, 618

Stanke, T., Schmidt, M. D., Gredel, R., \& Szokoly, G. 2002, A\&A, 393, 251

White, R. J., \& Basri, G. 2003, ApJ, 582, 1109

Yonekura, Y., Hayakawa, T., Mizuno, N., et al. 1999, PASJ, 51, 837 